\title{
Shot noise for resonant Cooper pair tunneling
}

\author{
Mahn-Soo Choi ${ }^{(1,2)}$, Francesco Plastina ${ }^{(3)}$, and Rosario Fazio ${ }^{(4)}$ \\ ${ }^{(1)}$ Department of Physics and Astronomy, University of Basel, Klingelberstrasse 82, 4056 Basel, Switzerland \\ (2) Korea Institute for Advanced Study, Cheonryanri-dong 207-43, Seoul 130-012, Korea \\ (3) NEST-INFM 83 Dipartimento di Scienze Fisiche ed Astronomiche, (DSFA) Università di Palermo, via Archirafi 36, \\ I-90123 Palermo, Italy \\ (4) NEST-INFM \& Dipartimento di Metodologie Fisiche e Chimiche (DMFCI), Università di Catania, viale A. Doria 6, \\ I-95125 Catania, Italy
}

(November 1, 2018)

\begin{abstract}
We study intrinsic noise of current in a superconducting single-electron transistor, taking into account both coherence effects and Coulomb interaction near a Cooper-pair resonance. Due to this interplay, the statistics of tunneling events deviates from the Poisson distribution and, more important, it shows even-odd asymmetry in the transmitted charge. The zero-frequency noise is suppressed significantly when the quasiparticle tunneling rates are comparable to the coherent oscillation frequency of Cooper pairs.
\end{abstract}

PACS Numbers: $72.70 .+\mathrm{m}, 73.23 \mathrm{Hk}, 74.50+\mathrm{r}$

Electron tunneling events across a small tunnel junction are correlated because of the large charging energy. These correlations lead to variety of phenomena which fall under the rubric of Coulomb blockade effects [1]. As an important example, the single-electron transistor (SET) has attracted much interest due to its ultimate sensitivity to electric charges [2]. If the junctions are superconducting, an additional effect, the coherent tunneling of Cooper pairs, comes to play and leads to much richer current-voltage characteristics [3., 4 .

Further understanding of the properties of electron transport (related, e.g., to coherence, electron-electron interaction and carrier statistics) comes from study of current fluctuations [5.66. In single-electron devices, the roles of Coulomb blockade on noise have been discussed by many authors 111 . Moreover the importance of coherence, leading to an enhancement of shot noise in superconducting quantum point contacts, was pointed out in Ref. [12]. Up to our knowledge, however, the combined effect of coherence and Coulomb blockade in superconducting double tunnel junction systems has not been addressed. Additional interest in studying noise in single-electron devices comes from their use in quantum measurements 13,14 and as entanglement detectors in solid state systems 15.

In this Letter, we discuss the statistics of tunneling events and the shot noise in superconducting SET near a resonance for Cooper pair tunneling. The interplay between coherence and interaction, explored by sweeping the device through the resonance, leads to a number of interesting results. i) At a Cooper pair resonance the statistics of tunneling events is non-Poissonian and it shows an even-odd asymmetry. ii) The shot noise suppression depends strongly on the ratio between the Josephson coupling and the quasi-particle tunneling rate (the effect is more pronounced close to the resonance). iii) The frequency-dependent noise has a resonance peak at a frequency corresponding to the coherent oscillation of Cooper pairs.

The superconducting SET [see Fig. 1 (a)] is a system of two small tunnel junctions in series with a small central electrode. The device operates in the regime in which the charging energy $E_{C}=e^{2} / 2 C_{\Sigma}\left(C_{\Sigma}\right.$ is the total capacitance of the island) is much larger than the Josephson coupling energy $E_{J}$ as well as the thermal energy $k_{B} T$. The largest energy scale is the superconducting gap $\Delta$ (assumed equal in both the electrodes and the island). By adjusting the bias and gate voltages, $V$ and $V_{g}$, one can put either the right or left junction at resonance for Cooper-pair tunneling [4, 16]. We consider the case of resonance across the left junction.

The effective Hamiltonian 17] is given by $H=H_{0}+$ $H_{q p}+H_{T}$ with 18]

$$
H_{0}=E_{C}\left(n+n_{0}\right)^{2}-e V n_{R}-E_{J} \cos 2 \phi_{L} .
$$

Here $n \equiv n_{L}+n_{R}$ is the number of excess electrons on the central island, $n_{L}\left(n_{R}\right)$ is the number of electrons that have passed across the left (right) junction into the central electrode, $e n_{0} \equiv C_{R} V+C_{g} V_{g}$ is the offset charge on the central island, and $2 \phi_{L}$ is the superconducting phase difference at the left junction. $n_{j}$ and $\phi_{j}$ are canonically conjugated $\left[\phi_{j}, n_{k}\right]=i \delta_{j k}$. The terms $H_{p q}$ and $H_{T}$ describe the quasiparticles on the electrodes and their tunneling across the junctions, respectively [팸]. They are given by

$$
\begin{gathered}
H_{q p}=\sum_{\alpha=L, R, D} \sum_{k \sigma} \varepsilon_{k \alpha} \gamma_{k \alpha \sigma}^{\dagger} \gamma_{k \alpha \sigma}, \\
H_{T}=\sum_{j=L, R} \sum_{k q \sigma}\left[T_{k q} e^{-i \phi_{j}} \gamma_{k j \sigma}^{\dagger} \gamma_{q D \sigma}+\text { h.c. }\right]
\end{gathered}
$$

where $\gamma_{k \alpha}^{\dagger}\left(\gamma_{k \alpha}\right)$ creates (annihilates) a quasiparticle with momentum $k$ and energy $\varepsilon_{k \alpha}=\sqrt{\xi_{k \alpha}^{2}+\Delta^{2}}$ in electrode 
$\alpha, \xi_{k}$ is the single-particle dispersion, and $T_{k q}$ is the tunneling amplitude. Each event of quasiparticle tunneling into (out of) the island across the junctions leads to the transition $n \rightarrow n+1(n \rightarrow n-1)$. The rate is given by

$$
\Gamma_{L / R}^{ \pm}(n)=\left[\operatorname{coth}\left(\beta \mathcal{E}_{n, \pm}^{L / R}\right) \pm 1\right] \frac{\operatorname{ImI}_{\mathrm{qp}}\left(\mathcal{E}_{\mathrm{n}, \pm}^{\mathrm{L} / \mathrm{R}}\right)}{2 e}
$$

where $\mathcal{E}_{n, \pm}^{L}= \pm E_{n, n \pm 1}, \mathcal{E}_{n, \pm}^{R}=e V \pm E_{n, n \pm 1}, E_{m, n}=$ $E_{C}(m-n)\left(m+n+2 n_{0}\right)$, and $I_{q p}$ is related to the quasiparticle tunneling current [19].

We will focus on the bias regime $|e V| \simeq 2 \Delta+E_{C}$ $\left(\gg E_{J}, k_{B} T\right)$ where two charge states, for example $n=0$ and $n=2$, are nearly degenerate. Then due to the strong Coulomb blockade, it suffices to keep the three charge states, $n=0,1,2$, and two tunneling rates, $\Gamma_{1} \equiv \Gamma_{R}^{-}(1)$ and $\Gamma_{2} \equiv \Gamma_{R}^{-}(2)$; the other tunneling rates are negligible. To simplify the notation, we will assume that $\Gamma_{1}=\Gamma_{2} \equiv \Gamma$, which is a very good approximation in the regime we are interested in. Effectively, one can imagine that across the left junction only coherent Cooper-pair tunneling occurs, interrupted from time to time by quasiparticle tunneling across the right junction, see Fig. 1 (b). In the experiment of Ref. [20], $1 / \Gamma_{1}=8 \mathrm{~ns}$ and $1 / \Gamma_{2}=6 \mathrm{~ns}$ for $E_{C}=2.3 E_{J}=117 \mu \mathrm{eV}$ and $\Delta=230 \mu \mathrm{eV}$.

We need to keep track of the variable $n_{R}$ (or alternatively $\left.n_{L}\right)$ as well as $n\left(n_{L}=n-n_{R}\right)$. Choosing the basis of $\left\{\left|n, n_{R}\right\rangle\right\}$, it can be shown that only diagonal elements (with respect to $n_{R}$ ) of the reduced density matrix are involved $\rho_{m n}\left(n_{R} ; t\right)=\left\langle m, n_{R}|\rho(t)| n, n_{R}\right\rangle$. The generalized master equation [4,21] can be written in the Lindblad form $(\hbar=1)$ :

$$
\partial_{t} \rho\left(n_{R}\right)=-i\left[H_{0}, \rho\left(n_{R}\right)\right]+\frac{1}{2} \sum_{n=1,2} \Gamma_{n}\left[2 L_{n} \rho\left(n_{R}+1\right) L_{n}^{\dagger}-L_{n}^{\dagger} L_{n} \rho\left(n_{R}\right)-\rho\left(n_{R}\right) L_{n}^{\dagger} L_{n}\right]
$$

where $L_{n}$ is a Lindblad operator corresponding to the quantum jump $n \rightarrow n-1, L_{n}=|n-1\rangle\langle n|$. The first term describes a purely phase-coherent dynamics, while the second is responsible for the dephasing and relaxation due to the quasiparticle tunneling.

Counting Statistics. We first investigate the statistical distribution of the number of electrons that have tunneled across the right junction. It has been obtained 22], first by defining the characteristic matrix $G(\theta, \tau)=\sum_{n_{R}} e^{-i \theta n_{R}} \rho\left(n_{R}, \tau+t\right)$, which satisfies a master equation similar to Eq. (5) with the initial condition $G(\theta, 0)=\sum_{n_{R}} \rho\left(n_{R}, t \rightarrow \infty\right)$. The probability $P(N, \tau)$ that $N$ electrons have tunneled during the period $\tau$ in the stationary state is then given by

$$
P(N, \tau)=\int_{-\pi}^{\pi} \frac{d \theta}{2 \pi} e^{+i \theta N} \operatorname{TrG}(\theta, \tau)
$$

When the dephasing is strong (either $\Gamma \gg E_{J}$ or $\left.\varepsilon \equiv E_{C}\left[\left(2+n_{0}\right)^{2}-n_{0}^{2}\right] \gg E_{J}\right)$, one can show that $(N<0)$

$$
\begin{gathered}
P(2 N, \tau)=\frac{1}{|N| !}\left(\frac{\Gamma_{r} \tau}{2}\right)^{|N|} \exp \left(-\frac{\Gamma_{r} \tau}{2}\right) \\
P(2 N-1, \tau)=0
\end{gathered}
$$

where $\Gamma_{r} \equiv 2 E_{J}^{2} \Gamma /\left(4 \varepsilon^{2}+\Gamma^{2}\right)$ is the relaxation rate for the charge state population in the strong dephasing limit. The distribution is Poissonian. However, there is a strong even-odd asymmetry. Physically, the charge is transferred in pairs (i.e. in units of $2 e$ ) rather than one by one.

In the weak dephasing limit $\left(\Gamma \ll E_{J}\right)$ at resonance $(\varepsilon=0)$, we find

$$
P(2 N, \tau)=\exp \left(-\frac{3 \Gamma \tau}{4}\right)\left(\frac{1}{3}+\frac{4}{\Gamma} \frac{\partial}{\partial \tau}\right) F_{|N|}(\tau)
$$

$$
P(2 N-1, \tau)=\frac{8}{3} \exp \left(-\frac{3 \Gamma \tau}{4}\right) F_{|N|}(\tau),
$$

where

$$
F_{n}(\tau)=\frac{1}{2 \pi i} \oint_{|z|=1} \frac{d z}{z^{n+1}} \frac{1}{\lambda(z)} \sinh \frac{\Gamma \tau \lambda(z)}{4}
$$

with $\lambda(z)=\sqrt{1+8 z}$. This distribution shows a much weaker, but still finite, even-odd asymmetry than the previous case [see Eq. (7) ]. In the long-time limit $(\Gamma \tau \rightarrow \infty)$, $P(2 N, \tau)=\frac{5}{9} P_{G}(N, \tau)$ and $P(2 N-1, \tau)=\frac{4}{9} P_{G}(N, \tau)$ where $P_{G}(N, \tau)$ is a Gaussian distribution with $\langle N\rangle=$ $I \tau / 2 e$ and $\left\langle(\Delta N)^{2}\right\rangle=20 \Gamma \tau / 27$.

In the intermediate case $\left(\Gamma \sim E_{J}\right)$, an analytic expression for $P(N, \tau)$ is not available. The numerical results are shown in Fig. 2. The distribution function deviates significantly from a Poissonian distribution function. Coherent oscillations of the Cooper pairs manifest themselves in the even-odd asymmetry of the transmitted charges: $P(N, \tau)$ is suppressed (enhanced) for odd (even) $N$ compared with the Poissonian distribution.

Shot Noise. The shot noise spectrum is defined as

$$
S(\omega)=\int_{-\infty}^{\infty} d \tau e^{i \omega \tau}\langle\{\delta I(t+\tau), \delta I(t)\}\rangle,
$$

where $\delta I(t)=I(t)-\langle I(t)\rangle$ and $\{A, B\}=A B+B A$. The total current $I(t)$ through the system is related to the tunneling currents $I_{L / R}=-e \partial_{t} n_{L / R}$ across the junctions by 10

$$
I(t)=\frac{C_{R}}{C_{\Sigma}} I_{L}(t)-\frac{C_{L}}{C_{\Sigma}} I_{R}(t) .
$$

It is convenient to define the spectral densities of tunneling currents $S_{i j}(\omega)(i, j=L, R)$ in an analogous way as in Eq.(10) and rewrite the noise power density in the form 


$$
S(\omega)=\frac{C_{R}^{2}}{C_{\Sigma}^{2}} S_{L L}(\omega)+\frac{C_{L}^{2}}{C_{\Sigma}^{2}} S_{R R}(\omega)-\frac{C_{L} C_{R}}{C_{\Sigma}^{2}}\left[S_{L R}(\omega)+S_{R L}(\omega)\right]
$$

In the stationary state $\langle I\rangle=\left\langle I_{L}\right\rangle=-\left\langle I_{R}\right\rangle$, so that $S(\omega)=S_{L L}(\omega)=S_{R R}(\omega)$ in the zero-frequency limit. In the opposite limit $(\omega \rightarrow \infty), S(\omega)=\left(C_{L}^{2} / C_{\Sigma}^{2}\right) S_{R R}(\omega)=$ $\left(C_{L}^{2} / C_{\Sigma}^{2}\right) 2 e\langle I\rangle$ [7,8,10]. [In our case, the left junction is (nearly) at resonance for the Cooper pair tunneling and hence $\lim _{\omega \rightarrow \infty} S_{L L}(\omega)=0$; see also the remarks below Eq. (四).]

In order to calculate the two-time correlators in Eq. (19), we follow the procedures based on the quantum regression theorem 21] starting from the master equation (5). An explicit (but lengthy) expression for $S(\omega)$ in terms of $\Gamma_{1,2}$ and $\varepsilon$ can be given at an arbitrary finite frequency [22]. Here we discuss the zero-frequency shot noise.

At $\omega=0$, the noise power density takes a simple form

$$
\frac{S(0)}{2 e I}=2-\frac{8\left(E_{J}^{2}+2 \Gamma^{2}\right) E_{J}^{2}}{\left(3 E_{J}^{2}+\Gamma^{2}+4 \varepsilon^{2}\right)^{2}} .
$$

In the strong dephasing limit $\left(\Gamma \gg E_{J}\right)$, the zerofrequency shot noise in Eq. (13) is enhanced approximately by a factor 2 compared with its classical value, $2 e I$. This can be understood in terms of Josephson quasiparticle (JQP) cycle [3,4]. Because of the fast quasiparticle tunneling across the right junction, each Cooper pair that has tunneled into the central island breaks up immediately into quasiparticles, and quickly tunnels out. The charge is therefore transfered in units of $2 e$ (compared with $e$ in classical charge transfer) for each JQP cycle [see also Eq. (7)].

In the weak $\left(\Gamma \ll E_{J}\right)$ and moderate $\left(\Gamma \simeq E_{J}\right)$ dephasing limits the semiclassical JQP picture breaks down. In the extreme case $\left(\Gamma \ll E_{J}\right)$, the quasipaticles do not see the left junction and consequently the system can be viewed (approximately) as a single-junction system. Still, the noise deviates slightly from the Poisson value since the channels for tunneling (i.e., $n=1 \rightarrow 0$ and $n=2 \rightarrow 1$ with corresponding rates $\Gamma_{1}$ and $\Gamma_{2}$ ) are correlated because of the Cooper pair oscillations and Coulomb blockade. The effect is related to the residual even-odd asymmetry of the distribution function in Eq. (8).

With moderate dephasing $\left(\Gamma \simeq E_{J}\right)$, quasiparticle tunneling events across the right junction are strongly affected by the coherent oscillation of Cooper pairs across the left junction. Indeed, this effect gives rise to the significant deviation from the Poissonian distribution of the tunneling statistics. More remarkably, it also leads to the suppression of the shot noise which is maximum (by factor $2 / 5)$ at resonance $(\varepsilon=0)$ for $\Gamma=\sqrt{2} E_{J}$, see Fig. 3. This is reminiscent of the shot noise suppression in (non-superconducting) double-junction systems
[8], whose maximal suppression is by factor $1 / 2$ for the symmetric junctions. We emphasize, however, that in the latter case, the coherence was not essential. In our case, the role of coherence is evident noticing that the dip in Fano factor [i.e., $S(0) / 2 e I$ ] disappears when moving away from the resonant condition as shown in Fig. 3 .

In Fig. 1 we show the typical behavior of the finitefrequency noise spectrum in the (a) strong and (b) weak dephasing limits. It is interesting to notice that (only) in the weak dephasing limit, there is a resonance peak at $\omega=E_{J}$. Near the maximum and for $\Gamma \ll E_{J}$, the noise behaves like 22]

$$
\frac{S(\omega)}{2 e I} \approx \frac{C_{R}^{2}}{2 C_{\Sigma}^{2}} \frac{E_{J}^{2}}{\left(\omega-E_{J}\right)^{2}+\Gamma^{2} / 4}
$$

The peak is an effect of coherent quantum transitions between the two energy levels tunnel split by $E_{J}$.

The JQP process discussed in this Letter was used in a recent experiment [20] to probe the coherent evolution of quantum states in a Cooper pair box. Weak continuous measurement using quantum point contact [23] and strong measurement using single-electron transistor 13. have been proposed. Whereas both schemes are noninvasive measurements, the setup discussed here probes the charge states on the island directly and invasively. In the weak dephasing limit, the resonance peaks in Eq. (14) and in Ref. [23] have a similar physical origin, and yet the latter has a peculiar upper bound. In the strong dephasing limit, the broad peak around zero frequency [Fig. A (a)] does not fit to the single Lorentzian shape of Ref. [13], which is a manifestation of the random telegraph noise; nevertheless, $\Gamma_{r}$ and $1 / \tau_{\text {mix }}$ in Ref. [13] give the same time scale describing the relaxation of population density of the charge states on the island.

In conclusion, we have investigated the combined effects of coherence and interaction on the statistics of tunneling events and the shot noise in a superconducting SET. It has been shown that the number distribution of tunneled electrons deviates from the classical Poisson distribution and that zero-frequency shot noise is suppressed significantly due the coherent oscillation of Cooper pairs in the presence of Coulomb blockade.

We express special thanks to E. Sukhorukov for many invaluable discussions. We also acknowledge useful discussions with Y. Blanter, C. Bruder, G. Falci, Y. Makhlin, A. Maassen van den Brink, G. Schön, and J. Siewert. 
[1] G. Schön and A.D. Zaikin, Phys. Rep. 198, 237 (1990); D.V. Averin and K.K. Likharev, Mesoscopic Phenomena in Solids, B.L. Altshuler, P.A. Lee, and R.A. Webb Eds., (North-Holland, Amsterdam, 1991).

[2] R. J. Schoelkopf et al., Science 280, 1238 (1998); A. N. Korotkov, Appl. Phys. Lett. 69, 2593 (1996).

[3] T. A. Fulton et al., Phys. Rev. Lett. 63, 1307 (1989).

[4] D. V. Averin and V. Y. Aleshkin, Pis'ma Zh. Eksp. Teor. Fiz. 50, 331 (1989) [JETP Lett. 50 (7), 367 (1989)]; A. Maassen van den Brink, G. Schön, and L. J. Geerligs, Phys. Rev. Lett. 67, 21 (1991).

[5] M. J. M. de Jong and C. W. J. Beenakker, in Ref. Mesoscopic Electron Transport, edited by L. L. Sohn, L. P. Kouwenhoven, and G. Schön (Kluwer Academic Publishers, Dordrecht, 1997).

[6] Y. M. Blanter and M. Büttiker, Phys. Rep. 336, 1 (2000).

[7] J. H. Davies et al., Phys. Rev. B 46, 9620 (1992).

[8] S. Hershfield et al., Phys. Rev. B 47, 1967 (1993)

[9] U. Hanke et al., Phys. Rev. B 48, 17209 (1993); U. Hanke, Y. M. Galperin, and K. A. Chao, ibid. 50, 1595 (1994).

[10] A. N. Korotkov, Phys. Rev. B 49, 10381 (1994).

[11] D. V. Averin, cond-mat/0010052; E. V. Sukhorukov, G. Burkard, D. Loss, cond-mat/0010458.

[12] D.V. Averin and H. T. Imam, Phys. Rev. Lett. 76, 3814 (1996).

[13] Yu. Makhlin, G. Schön, and A. Shnirman, Phys. Rev. Lett. 85, 4578 (2000).

[14] M. H. Devoret and R. J. Schoelkopf, Nature 406, 1039 (2000).

[15] D. Loss and E.V. Sukhorukov, Phys. Rev. Lett. 84, 1035 (2000); M.-S. Choi, C. Bruder, and D. Loss, Phys. Rev. B 62, 13569 (2000).

[16] M.-S. Choi et al., Europhys. Lett.53, 251 (2001).

[17] A microscopic derivation is given in G.-L. Ingold and Y. V. Nazarov, in Single Charge Tunneling: Coulomb Blockade Phenomena in Nanostructures, edited by $\mathrm{H}$. Grabert and M. Devoret (Plenum Press, New York, 1992).

[18] In Eq. (1), we have neglected the Josephson term, $-E_{J} \cos \phi_{R}$, associated with the right junction in the spirit of the rotating wave approximation; see, e.g., K. Blum, Density Matrix Theory and Applications, 2 ed. (Plenum Press, New York, 1996).

[19] N. R. Werthamer, Phys. Rev. 147, 255 (1966); A. I. Larkin and Y. N. Ovchinnikov, Zh. Eksp. Teor. Fiz. 51, 1535 (1966) [Sov. Phys. -JETP 24, 1035 (1967)].

[20] Y. Nakamura, Y. A. Pashkin, and J. S. Tsai, Nature 398, 786 (1999).

[21] H. J. Carmichael, An Open Systems Approach to Quantum Optics (Springer-Verlag, Berlin, 1993).

[22] M.-S. Choi, F. Plastina, and R. Fazio, in preparation.

[23] A. N. Korotkov, Phys. Rev. B. 60, 5737 (1999); A. N. Korotkov and D. V. Averin, cond-mat/0002203.
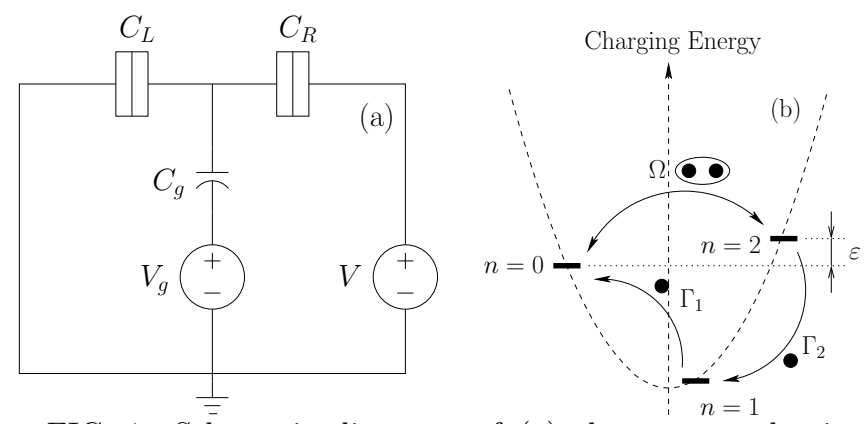

FIG. 1. Schematic diagrams of (a) the superconducting SET device and (b) the transition processes between relevant charge states.

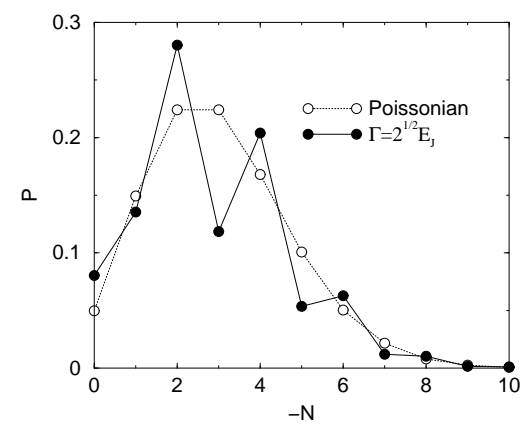

FIG. 2. Probability distribution function $P(N, \tau)$ at $\Gamma \tau=4$ for $\Gamma=\sqrt{2} E_{J}$ (solid line). For a comparison, the Poissonian distribution is also plotted (dotted line). Notice that $N<0$ by definition.

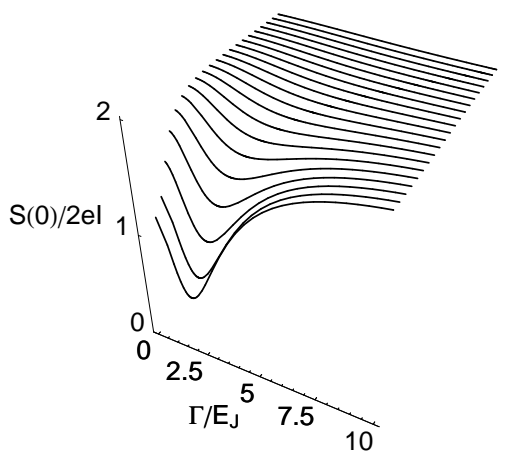

FIG. 3. Normalized zero-frequency shot noise for $\varepsilon / E_{J}=0,0.25, \cdots, 5$. The dip in the noise is most pronounced at resonance $\left(\varepsilon / E_{J}=0\right)$.
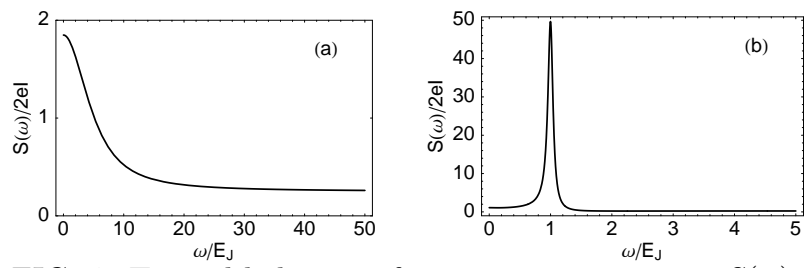

FIG. 4. Typical behavior of noise power spectrum $S(\omega)$ as a function of frequency $\omega$ in the (a) strong $\left(\Gamma_{1,2} \gg E_{J}\right)$ and (b) weak $\left(\Gamma_{1,2} \ll E_{J}\right)$ quasi-particle tunneling limits. For 